\title{
PERFORMANCE OF THE INTEGRATED TRACKER TOWERS OF THE GLAST LARGE AREA TELESCOPE
}

\author{
M. BRIGIDA, A. CALIANDRO, C. FAVUZZI, P. FUSCO, F. GARGANO, \\ N. GIGLIETTO, F. GIORDANO, F. LOPARCO, B. MARANGELLI, \\ M. N. MAZZIOTTA, N. MIRIZZI, S. RAINÒ AND P. SPINELLI \\ for the GLAST LAT Collaboration \\ Dipartimento Interateneo di Fisica "M. Merlin" and INFN Bari \\ Via E. Orabona 4, \\ 70126 Bari, Italy \\ E-mail: francesco.loparco@ba.infn.it
}

\begin{abstract}
The GLAST Large Area Telescope (LAT) is a high energy gamma ray observatory, mounted on a satellite that will be flown in 2007. The LAT tracker consists of an array of tower modules, equipped with planes of silicon strip detectors (SSDs) interleaved with tungsten converter layers. Photon detection is based on the pair conversion process; silicon strip detectors will reconstruct tracks of electrons and positrons. The instrument is actually being assembled. The first towers have been already tested and integrated at Stanford Linear Accelerator Center (SLAC). An overview of the integration stages of the main components of the tracker and a description of the pre-launch tests will be given. Experimental results on the performance of the tracker towers will be also discussed.
\end{abstract}

\section{The Tracker of the GLAST Large Area Telescope}

The GLAST mission will study the gamma-ray sky in the energy range from $1 \mathrm{keV}$ to $300 \mathrm{GeV}$, allowing the investigation of many fields of the gammaray astrophysics. The GLAST satellite will be instrumented with two main detectors: the Large Area Telescope (LAT), that will operate in the energy range from $20 \mathrm{MeV}$ to $300 \mathrm{GeV}$, and the GLAST Burst Monitor (GBM), that will operate in the range from $1 \mathrm{keV}$ to $30 \mathrm{MeV}$.

The LAT is a high energy pair conversion telescope. It will consist of three subsystems: a precision silicon strip detector (SSD) tracker (TKR), a CsI calorimeter (CAL), and a plastic scintillator anticoincidence detector $(\mathrm{ACD})$. The LAT design is modular, an is composed by an array of $4 \times$ 4 identical towers, supported by a low-mass grid structure. Each tower 
includes a TKR module, a CAL module and a DAQ unit. The tower array is surrounded by the segmented plastic scintillators composing the ACD.

Each TKR tower has a $37.2 \times 37.2 \mathrm{~cm}^{2}$ cross section and it is $75 \mathrm{~cm}$ high. It is composed of layers of SSDs and thin tungsten converter foils. The $e^{+}-e^{-}$pairs produced by photons interacting in the conversion foils are tracked by the SSDs and their energy is mesured by the calorimeter downstream. The SSDs and the converter foils are arranged in a structure consisting of a stack of 19 panels, called trays. Each tray holds a converter foil and two of SSD layers. The upper 12 trays are equipped with a $3 \% X_{0}$ converter foil, the middle 4 trays with a $18 \% X_{0}$ converter and bottom 3 trays without converter foils.

The active detectors are $400 \mu \mathrm{m}$ thick single-sided SSD wafers with a cross section of $8.95 \times 8.95 \mathrm{~cm}^{2}$, bonded together into $35.8 \mathrm{~cm} \times 8.95 \mathrm{~cm}$ ladders. The strip pitch is of $228 \mu \mathrm{m}$ and there are 384 strips per ladder ${ }^{1}$. The TKR read-out electronics encompasses about one milion of strips and amplifier-discriminator channels. The current signal from each strip is converted into a voltage pulse by a charge-sensitive preamplifier coupled with a shaper. Finally, the shaper output signal is converted into a digital signal by a discriminator. The discrimination threshold corresponds to about $1 / 4$ of the pulse amplitude induced by a minimum ionizing particle.

For each event, the readout electronics provides a digital output that includes two kinds of information: the list of the fired strips, i.e. of the channels whose signal amplitude exceeds the threshold, and a set of Time over Threshold (ToT) values, each corresponding to a single SSD layer. The ToT represents the time interval during which, at least a signal from a strip belonging to the plane, exceeds the threshold. The information about the fired strips is used for tracking purpose. On the other hand, since the ToT is almost proportional to the energy deposited inside the SSDs, the ToT information can be used to estimate the number of particles crossing a SSD planes, In particular, in gamma-ray events, the ToT information can be used to search the position of the photon conversion vertex.

\section{Integration of the LAT Tracker}

The construction of the LAT TKR is responsibility of the Italian INFN collaboration involved in the project. After being assembled in the INFN laboratories, the TKR towers are delivered to the Alenia test facility in Rome, where both vibration and thermal-vacuum tests are performed. During the environmental testing, functional tests are also performed on the 
TKR towers. After the environmental tests, TKR towers are shipped to SLAC, where they are integrated on the grid structure together with the CAL modules. Actually, both the construction and testing of the TKR towers have been completed, and all towers have been delivered to SLAC for integration.

Before being integrated on the grid, TKR towers are tested with cosmic rays. Table 1 shows the average efficiencies of the SSD layers composing the TKR towers delivered to SLAC. All TKR towers exhibit an average efficiency greater than 99\%, with the exception of the first tower (labelled as tower "A"), that shows a slightly lower efficiency. Actually, 14 towers have already been integrated, and the remaining towers are ready to be installed.

Table 1. Average efficiencies of the SSDs composing the TKR towers delivered to SLAC.

\begin{tabular}{||l||c|c|c|c|c|c|c|c||}
\hline TKR ID & $\mathrm{A}$ & $\mathrm{B}$ & 1 & 2 & 3 & 4 & 5 & 6 \\
\hline Efficiency (\%) & 98.59 & 99.59 & 99.51 & 99.62 & 99.43 & 99.62 & 99.68 & 99.61 \\
\hline \hline TKR ID & 7 & 9 & 10 & 11 & 13 & 12 & 14 & 8 \\
\hline Efficiency (\%) & 99.68 & 99.69 & 99.67 & 99.68 & 99.74 & 99.68 & 99.68 & 99.77 \\
\hline
\end{tabular}

\section{Study of the performance of the LAT TKR towers}

To study the performance of the integrated LAT TKR towers, we have analyzed the cosmic ray data samples. The present analysis has been performed using the data samples obtained in the 8-tower configuration. Further data samples are being taken during the stages of the LAT integration.

For or analysis we selected a data sample consisting of single muon events fully contained in a single TKR tower. The TKR trigger condition requires the coincidence among 3 consecutive SSD planes in both $x$ and $y$ views. Hence, the selected muon tracks have to cross at least 6 SSD layers. About $2 \times 10^{6}$ events survived to these cuts.

\subsection{Study of the ToT in SSD layers}

Figure 1 shows the distribution of the ToTs in the SSD layers crossed by muon tracks.

We have also examined the dependence of the ToT in the track layers on the geometrical parameters describing muon tracks, i.e. the zenith angle $\theta$ and the azimuth angle $\phi$.

Figure 2(a) shows the average ToT as a function of $1 / \cos \theta$. As could be expected, the average ToT increases linearly with $1 / \cos \theta$. As discussed 


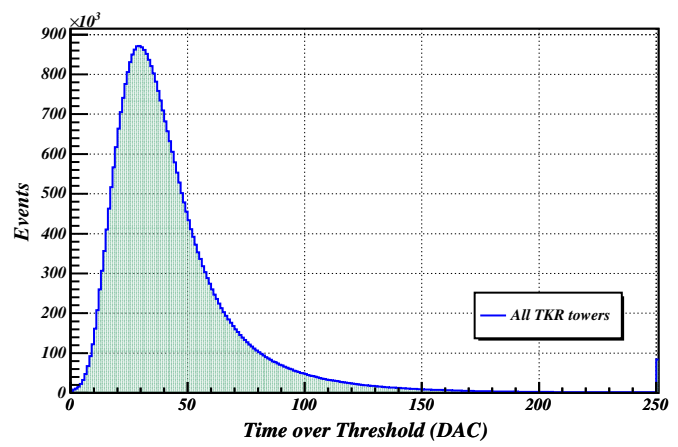

Figure 1. Distribution of the ToT in the SSD layers crossed by muon tracks. A DAC unit corresponds to $200 \mathrm{~ns}$.

in section 1, the ToT is proportional to the energy deposited in SSD layers, that is proportional to the track length. Hence, the ToT has to be depend linearly to the track length, that is proportional to $1 / \cos \theta$.

The dependence of the ToT on the azimuth angle $\phi$ is more complicated, because SSD strips are oriented along two orthogonal direction. For tracks inducing signals on groups of strips, the ToT will be proportional to the maximum pulse amplitude. A useful physical quantity that can be introduced is the projected track length, $l^{\prime}$, that is the projection of the track length $l$ along the strip view:

$$
l^{\prime}=\left\{\begin{array}{l}
l \sqrt{\cos ^{2} \theta+\sin ^{2} \theta \cos ^{2} \phi} \text { for } \mathrm{x} \text {-view layers } \\
l \sqrt{\cos ^{2} \theta+\sin ^{2} \theta \sin ^{2} \phi} \text { for } \mathrm{y} \text {-view layers }
\end{array}\right.
$$

Figure 2(b) shows that the ToT increases linearly with the ratio $l / l^{\prime}$.

\subsection{ToT in triggering layers: evaluation of the hit capture efficiency}

After a trigger is being issued, a trigger acknowledgment signal (TACK) is generated, that takes a time of about $2 \mu s$ to be sent to the readout chips. If the pulse amplitudes are still over the threshold when the chips are reached by the TACK signal, the corresponding hits are captured. On the other hand, if the pulses are below the threshold, the hits are lost and a null ToT is recorded.

The study of ToT in triggering layers is useful because it allows to evaluate the hit capture efficiency. To generate a trigger, a 6 -fold coincidence is required among 3 consecutive pairs of $\mathrm{x}$-view and $\mathrm{y}$-view layers. Hence, 

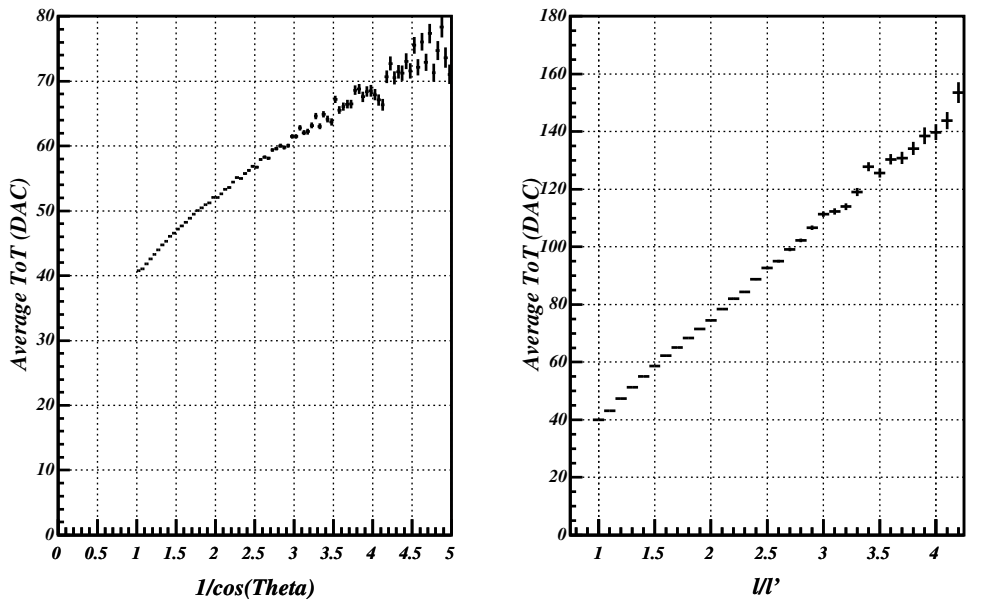

Figure 2. Average ToT in the SSD layers crossed by muon tracks as a function of the track parameters: (a) ToT as a function of the inverse cosine of the zenith angle; (b) ToT as a function of the ratio between the track length and the projected track length along the strip view.

the probability of noisy layers being involved in the trigger is negligible. The hit capture unefficiency $1-\epsilon$ is evaluated as the fraction of events with null ToT. From table 2 it is evident that the hit capture unefficiencies are always less than $10^{-3}$, except for the tower "A", that has been mounted on the bay 0 .

Table 2. Hit capture unefficiencies of the first 8 integrated TKR towers.

\begin{tabular}{||l||c|c|c|c|c|c|c|c||}
\hline Bay ID & 0 & 1 & 4 & 5 & 8 & 9 & 12 & 13 \\
\hline$(1-\epsilon) \times 10^{4}$ & 32 & 8.2 & 7.9 & 8.1 & 8.0 & 9.1 & 6.4 & 5.4 \\
\hline
\end{tabular}

\section{Conclusions}

The construction and testing of the LAT TKR towers has been completed and their integration is currently in progress. We have performed an analysis of the cosmic ray data collected by the first 8 integrated towers, that shows that the TKR behaviour is consistent with expectations.

\section{References}

1. GLAST- Scientific and Technical Plane, AO 99-OSS-03 